\title{
Esófago negro secundario a esofagitis necrotizante aguda. Reporte de un caso clínico
}

\author{
Martin E W allberg'1,4, Pablo Young'2, Bárbara C Finn², \\ Marcelo Thomé ${ }^{3}$, Andrea A Chueco ${ }^{1,4}$, Fernando Villarejo ${ }^{4}$. \\ Black esophagus due to acute \\ necrotizing esophagitis. \\ Report of one case
}

Acute esophageal necrosis, which presents as a black esophagus on endoscopy, is an uncommon occurrence. It is defined as a dark pigmentation of the esophagus associated with histologic mucosal necrosis. We report a 75-year-old men who developed black esophagus due to acute esophageal necrosis caused by septicemia. The patient had a favorable evolution (Rev Méd Chile 2009; 137: 672-4).

(Key Words: Endoscopy, digestive system; Esophagitis; Sepsis)

Recibido el 26 de agosto, 2008. Aceptado el 28 de noviembre, 2008.

Servicios de ${ }^{1}$ Emergencias, ${ }^{2}$ Clínica Médica, Hospital Británico de Buenos Aires;

${ }^{3}$ Gastroenterología y ${ }^{4}$ Unidad de Terapia Intensiva, Clínica Olivos, Argentina.

$\mathrm{E}^{\mathrm{s}}$ esófago negro se caracteriza por la presencia de pigmentación negra difusa del esófago, que en el caso de la esofagitis necrotizante (EN) se acompaña de necrosis de la mucosa esofágica ${ }^{1-3}$. La necrosis casi siempre es circunferencial, puede extenderse a lo largo de todo el esófago, y termina abruptamente en la unión gastroesofági$\mathrm{ca}^{2-5}$, siempre en ausencia de ingesta de cáusticos 0 agentes corrosivos ${ }^{2-6}$. Fue descrita por Brennan en 1967 en una autopsia y posteriormente en endoscopia por Goldenberg y $\mathrm{col}^{7}$, en 1990. Se han descrito hasta la actualidad 100 pacientes en la literatura. Presentamos el caso de un paciente con diagnóstico de esófago negro debido a EN secundaria a shock séptico por bacilos Gram negativos.

Correspondencia a: Dr. Pablo Young. Hospital Británico. Perdiel 74 (1280) Buenos Aires, Argentina. Tel 541143096400. Fax 5411 43043393. E mail: pabloyoung2003@yahoo.com.ar

\section{CASO CĹNICO}

Paciente de sexo masculino de 75 años con antecedentes de hipertensión arterial que ingresó a la Unidad de Cuidados Intensivos con el diagnóstico de shock. Presentó vómitos alimentarios y pirosis durante las veinticuatro horas previas al ingreso. La familia refinó un episodio de fiebre y escalofríos. Se colocó una sonda nasogástrica observándose al aspirar un contenido en borra de café. No refeńa hematemesis o melena. El paciente fue reanimado con infusión de cristaloides y requirió drogas vasopresoras durante las primeras $24 \mathrm{~h}$ (noradrenalina $0,1 \mathrm{mcg} / \mathrm{kg} / \mathrm{min}$ ). A su ingreso se realizaron hemocultivos y urocultivo, ambos fueron positivos a las $48 \mathrm{~h}$ para Escherichia coli multisensible. Se inició tratamiento empírico con ceftriaxona $1 \mathrm{gr}$ cada $12 \mathrm{~h}$ que luego se cambió según sensibilidad y cumplió tratamiento antibiótico por 14 días. La analítica demostró leucocitosis y anemia con hematocrito de $30 \%$.

A las doce horas del ingreso, ya estabilizado el paciente, se realizó videoendoscopia digestiva alta (VEDA) en donde se observó en esófago mucosa 


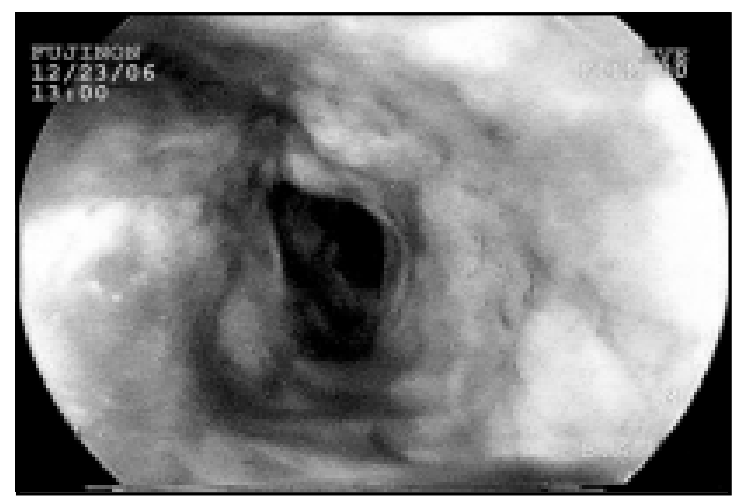

Figura 1A. Video endoscopia digestiva alta: necrosis esofágica extensa de color negro.

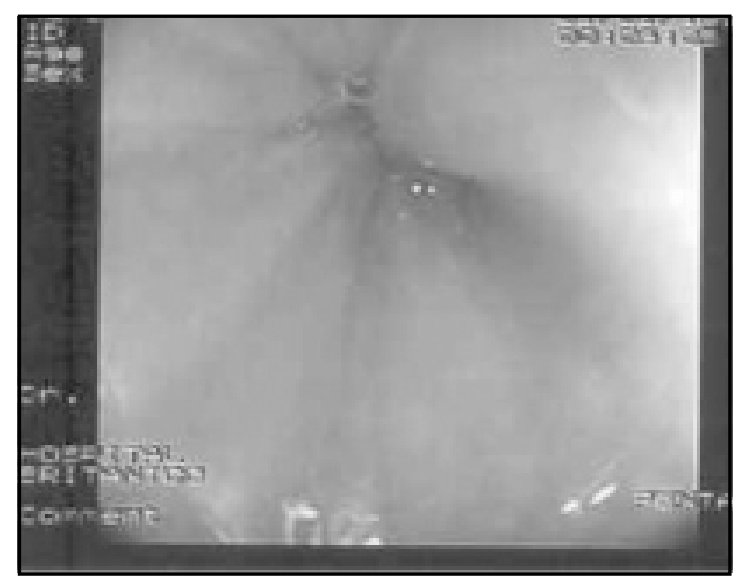

Figura 1B. Esófago Normal.

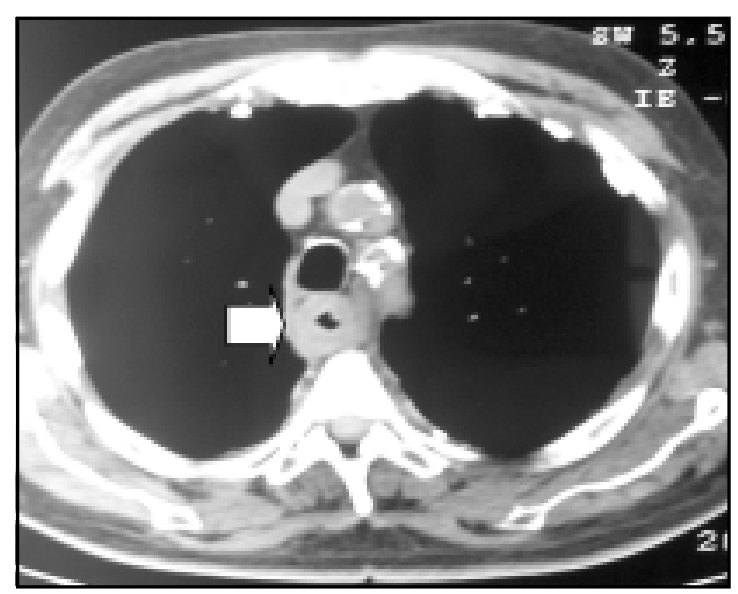

Figura 2. TAC de tórax: demuestra engrosamiento del esófago (flecha blanca). necrótica, con áreas de esfacelo; en estómago erosiones aisladas en antro y restos hemáticos (Figura 1A). La biopsia confirmó la EN. No presentó antecedentes de ingesta de cáusticos ni sustancias corrosivas. Se compara la imagen previa con una VEDA normal (Figura 1B).

Se administraron drogas inhibidoras de la bomba de protones por vía endovenosa, sucralfato por vía oral y se agregó al tratamiento antibiótico previo, metronidazol $500 \mathrm{mg} /$ cada $8 \mathrm{~h}$ por siete días.

Se realizó tomografía axial computada de tórax (TAC) que mostró engrosamiento de la pared del esófago a predominio mucoso en toda su extensión (Figura 2). A los tres días del ingreso se realizó una radiografía de esófago-estómago-duodeno que mostró buen pasaje esofágico, reflujo gastroesofágico en tercio inferior del esófago con mucosa irregular y pliegues gruesos. No hubo fuga de contraste.

Inició ingesta alimentaria a las $96 \mathrm{~h}$, con buena tolerancia. Evolucionó en forma favorable por lo cual egresó. Se repitió la VEDA al mes del egreso, siendo ésta normal.

\section{Discusión}

El esófago negro se caracteriza por la presencia de pigmentación negra difusa del esófago, que en el caso de la EN se acompaña de necrosis de la mucosa $^{1-10}$. La EN es una entidad muy rara. Ben Soussan y $\mathrm{col}^{2}$ describen una incidencia de $0,2 \%$ (8 casos en 3.900 endoscopias), mientras que Grudell y $\mathrm{col}^{8}$ reportan una incidencia de 0,008\% (6 casos en 78.847 endoscopias).

Gurvits y $\mathrm{col}^{10}$ analizan la literatura hasta el año 2006, encontrando un total de 88 pacientes con EN, 70 correspondieron al sexo masculino y 16 al femenino con una edad promedio de 67 años.

Esta patología generalmente se presenta en el adulto y en aquellos con enfermedades asociadas, tales como cáncer, insuficiencia renal, enfermedad coronaria o vascular periférica, diabetes, alcoholismo y cirrosis ${ }^{1-11}$. También se ha descrito su asociación con candidiasis, esofagitis herpética, cetoacidosis diabética, síndrome antifosfolipídico, shock, hipotermia, vómitos abundantes postingesta de alcohol, vólvulo gástrico, síndrome pilórico, trauma por sonda nasogástrica e hipersensibilidad a fármacos (síndrome de Steven-Johnson) ${ }^{12-17}$. 
La EN es una condición patológica probablemente relacionada a una injuria isquémica ${ }^{7,13,18}$, ya que la rápida resolución de la lesión después de la estabilización hemodinámica sugiere que la reducción temporaria de la perfusión puede resultar en la necrosis de la mucosa esofágica $2,3,5$. Otra teońa es que el íleo transitorio, con acumulación de grandes cantidades de jugo gástrico, y prolongado reflujo gastroesofágico, lesiona la mucosa al sobrepasar los mecanismos de defensa, lo que además se ve amplificado en los casos de bajo gasto cardíaco ${ }^{2}$.

La presentación clínica más común es el sangrado digestivo alto, hematemesis, vómitos en borra de café o melena en $70 \%$ de los casos, aunque puede haber otros síntomas como dolor epigástrico, náuseas, vómitos, disfagia, o shock 2,8,17-19.

El diagnóstico de esófago negro por EN se realiza con endoscopia ${ }^{2}$, debiendo diferenciarse de otras entidades por medio de la biopsia, como melanosis, pseudomelanosis, melanoma, Acantosis nigricans, exposición a polvo de carbón, intoxicación con tinturas, y ocronosis ${ }^{2-19}$. Los diagnósticos diferenciales de EN isquémica son la necrosis local secundaria a

\section{REFERENCIAS}

1. Cattan P, Cullerier E, Celuer C, Carnot F, Landi B, DusoleIl A, BARBIER JP. Black esophagus associated with herpes esophagitis. Gastrointest Endosc 1999; 49: 105-7.

2. Ben Soussan E, Savoye G, Hochain P, Hervé S, Antonietti M, Lemoine F, Ducrotté P. Acute esophageal necrosis: a 1-year prospective study. Gastrointest Endosc 2002; 56: 213-7.

3. Katsinelos P, Pilpilidis I, Dimiropoulos S, Paroutoglou $\mathrm{G}$, Kamperis E, TSOLKas P ET AL. Black esophagus induced by severe vomiting in a healthy young man. Surg Endosc 2003; 17: 521.

4. Pantanowitz L, Gelrud A, Nasser I. Black esophagus. Ear Nose Throat J 2003; 82: 450-2.

5. Reichart M, Busch ORC, Bruno MJ, van Lanschot JJB. Black esophagus: a view in the dark. Dis Esophagus 2000; 13: 311-3.

6. Hwang J, Weigel TL. Acute Esophageal Necrosis: “Black Esophagus”. JSLS 2007; 11: 165-7.

7. Goldenberg SP, Wain SL, Marignani P. Acute Necrotizing Esophagitis. Gastroenterology 1990; 96: 493-6.

8. GRudell ABM, Mueuer PS, Viggiano TR. Black esophagus: report of six cases and review of the literatura, 1963-2003. Dis Esophagus 2006; 19: 105-10.

9. Khan AM, Hundal R, Ramaswamy V, Korsten M, Dhuper S. Acute esophageal necrosis and liver pathology, a rare combination. World J Gastroenterol 2004; 10: 2457-8.

10. Gurvits GE, Shapsis A, Lau N, Gualtieri N, Robilotti infecciones, el hematoma intramural del esófago, la injuria por cáusticos, el síndrome pilónico con reflujo severo, necrosis inducida por antibióticos de amplio espectro y el síndrome de Steven-Johnson.

El tratamiento debe estar dirigido a reanimar el estado hemodinámico optimizando la perfusión, con restricción de la ingesta oral, nutrición parenteral, inhibición de la secreción ácida, tratamiento del reflujo, antibióticos y solución de la causa de base $^{2-19}$. En algunos casos se requiere cirugía con interposición colónica ${ }^{3}$.

El pronóstico varía, pero la mayoría se recuperan con manejo conservador ${ }^{3-9}$, entre las complicaciones, que aparecen hasta en $25 \%$ de los pacientes, se observan la estenosis esofágica, que puede requerir repetidas dilataciones y la perforación esofágica con o sin absceso o mediastinitis ${ }^{12}$.

La mortalidad puede llegar a 30\%, aunque por lo general depende más de la enfermedad subyacente que de la $\mathrm{EN}^{3,6,12}$.

Como conclusión creemos que es necesario el conocer de esta entidad para minimizar la solicitud de estudios y de inicio mantener un manejo conservador.

JG. Acute esophageal necrosis: a rare syndrome. J Gastroenterol 2007; 42: 29-38.

11. Tsokos M, HeRBST H. Black oesophagus: a rare disorder with potentially fatal outcome. A forensic pathological approach based on five autopsy cases. Int J Legal Med 2005; 119: 146-52.

12. KІм YH, Cног SY. Black esophagus with concomitant candidiasis developed after diabetic ketoacidosis. World J Gastroenterol 2007; 13: 5662-3.

13. Haviv YS, Reinus C, Zimmerman J. Black Esophagus: A rare complication of shock. Am J Gastroenterol 1996; 91: 2432-4.

14. Cadot P, Duverger V, Imperato M, Lapprand M, Vergos M. Black esophagus associated with hypothermia. Ann Chir 2001; 126: 903-5.

15. Kram M, Gorenstein L, Eisen D, Cohen D. Acute esophageal necrosis associated with gastric volvulus. Gastrointest Endosc 2000; 51: 610-2.

16. Mangan T, Thompson Coley A, Wytock D. Antibiotic associated acute necrotizing esophagitis. Gastroenterology 1990; 99: 900 (Letter).

17. Gejer A, Aguilar H, Burgart L, Gostout CJ. The black esophagus. Am J Gastroenterol 1995; 12: 2210-2.

18. Caselia G, Perego D, Corti G, Cambareri AR, Buda CA, ZoLdAN C, BALDINI V. Black esophagus: Should it be considered an unfavorable prognostic factor? Dis Esophagus 2001; 14: 166-8.

19. Obermeyer R, Kasirajan K, Erzurum V, Chung D. Necrotizing esophagitis presenting as a black esophagus. Surg Endosc 1998; 12: 1430-3. 